\title{
Convergence Rates of Density Estimation in Besov Spaces
}

\author{
Huiying Wang \\ Department of Applied Mathematics, Beijing University of Technology, Beijing, China \\ E-mail:b200806005@emails.bjut.edu.cn \\ Received July 29, 2011; revised August 23, 2011; accepted August 30, 2011
}

\begin{abstract}
The optimality of a density estimation on Besov spaces $B_{r, q}^{s}(\boldsymbol{R})$ for the $L_{p}$ risk was established by Donoho, Johnstone, Kerkyacharian and Picard ("Density estimation by wavelet thresholding," The Annals of Statistics, Vol. 24, No. 2, 1996, pp. 508-539). To show the lower bound of optimal rates of convergence $R_{n}\left(B_{r, q}^{s}, p\right)$, they use Korostelev and Assouad lemmas. However, the conditions of those two lemmas are difficult to be verified. This paper aims to give another proof for that bound by using Fano's Lemma, which looks a little simpler. In addition, our method can be used in many other statistical models for lower bounds of estimations.
\end{abstract}

Keywords: Optimal Rate of Convergence, Density Estimation, Besov Spaces, Wavelets

\section{Introduction}

Wavelet analysis has many applications, one of which is to estimate an unknown density function based on independent and identically distributed (i.i.d.) random samples. Let $(\Omega, \aleph, P)$ be a probability measurable space and $X_{1}, \cdots, X_{n}$ be i.i.d. random variables with an unknown density function $f$. We use $E(X)$ to denote the expectation of a random variable $X$. The sequence $R_{n}(V, p):=\inf _{f_{n}} \sup _{f \in V} E\left(\left\|f_{n}-f\right\|_{p}\right)$ is called optimal rate of convergence on the functional class $V$ for the $L_{p}$ risk. Here, $f_{n}$ is an arbitrary estimator of $f$ with $n$ i.i.d. random samples. Kerkyacharian and Picard [1] study $R_{n}(V, p)$ when $V$ is a Besov space with matched case. Donoho, Johnstone, Kerkyacharian and Picard [2] consider unmatched cases. In fact, they show the optimal convergence rates for $L_{r}$ Besov class $\tilde{B}_{r, q}^{s}$ and $L_{p}$ risk

$$
R_{n}\left(\tilde{B}_{r, q}^{s}, p\right) \sim\left\{\begin{array}{cc}
(\ln n / n)^{\frac{s-1 / r+1 / p}{2(s-1 / r)+1}}, & r \leq \frac{p}{2 s+1}, \\
n^{-\frac{s}{2 s+1}}, & r>\frac{p}{2 s+1} .
\end{array}\right.
$$

To show the lower bound of (1.1), authors of [2,3] use Korostelev and Assouad lemmas. However, the conditions of those two lemmas are difficult to be verified. In this small paper, we give another proof for the lower bound of (1.1) by using Fano's lemma [4]. It should be pointed out that Fano's lemma can be used to a variety of statistical models, see [5-7].

As usual, $L_{p}(R)(p \geq 1)$ denotes the classical Lebesgue space on the real line $R$. In particular, $L_{2}(R)$ stands for the Hilbert space, which consists of all square integrable functions. As a subspace of $L_{p}(R)$, the Sobolev space with an integer exponent $k$ means

$$
W_{p}^{k}(R):=\left\{f, f^{(m)} \in L_{p}(R), m=0,1, \cdots, k\right\}(p \geq 1) .
$$

The corresponding norm

$$
\|f\|_{W_{p}^{k}}:=\|f\|_{p}+\left\|f^{(k)}\right\|_{p} .
$$

Moreover, the Besov space $B_{p, q}^{s}(R)$ [3] $(1 \leq p, q \leq \infty$, $s=n+\alpha$ and $\alpha \in(0,1])$ can be defined by

$$
B_{p, q}^{s}(R)=\left\{f \in W_{p}^{n}(R),\left(2^{j \alpha} \omega_{p}^{2}\left(f^{(n)}, 2^{-j}\right)\right)_{j \in Z} \in l_{q}\right\}
$$

with the associated norm

$$
\|f\|_{B_{p, q}^{s},}:=\|f\|_{W_{p}^{n}}+\left\|\left\{2^{j \alpha} \omega_{p}^{2}\left(f^{(n)}, 2^{-j}\right)\right\}\right\|_{l_{q}(Z)},
$$

where

$$
\omega_{p}^{2}(f, t):=\sup _{|h| \leq t}\|f(x+2 h)-2 f(x+h)+f(x)\|_{p} .
$$

In general, it can be shown that compactly supported and $n$ times differentiable functions belong to $B_{p, q}^{s}(R)$ for 
$0<s<n$ and $1 \leq p, q \leq \infty$.

The Besov space can be discretized by the sequence norm of wavelet coefficients. Many useful wavelets are generated by scaling functions. More precisely, if $\varphi$ is a scaling function with

$$
\varphi(x)=\sum_{k} h_{k} \sqrt{2} \varphi(2 x-k),
$$

then $\psi(x):=\sum_{k}(-1)^{k} h_{1-k} \sqrt{2} \varphi(2 x-k)$ defines a wavelet [3]. Clearly, when $\varphi$ is compactly supported and continuous, the corresponding wavelet $\psi$ has the same properties. An orthonormal wavelet basis of $L_{2}(R)$ is generated from dilation and translation of a scaling function and its corresponding wavelet, i.e.

$$
\begin{aligned}
\left\{\varphi_{j_{0} k}(x)\right. & :=2^{\frac{j_{0}}{2}} \varphi\left(2^{j_{0}} x-k\right), \\
\psi_{j k}(x) & \left.:=2^{\frac{j}{2}} \psi\left(2^{j} x-k\right)\right\}_{j \geq j_{0}, k \in Z} .
\end{aligned}
$$

Although wavelet basis are constructed for $L_{2}(R)$, most of them constitute unconditional bases for $L_{p}(R)$. A scaling function $\varphi$ is called $t$ regular, if $\varphi$ has continuous derivatives of order $t$ and its corresponding wavelet $\psi$ has vanishing moments of order $t$, i.e.

$$
\int x^{k} \psi(x) \mathrm{d} x=0, k=0,1, \cdots, t-1 .
$$

The following lemma [3] plays important roles in this paper.

Lemma 1.1. Let $\varphi$ be a compactly supported, $t$ regular orthonormal scaling function with the corresponding wavelet $\psi$ and $0<s<t$. If $f \in L_{p}(R), s_{0 k}:=\left\langle f, \varphi_{0 k}\right\rangle$, $d_{j k}:=\left\langle f, \psi_{j k}\right\rangle$ and $1 \leq p, q \leq \infty$, then the following two conditions are equivalent:

1) $f \in B_{p, q}^{s}(R)$;

2) $\left\|S_{0} \cdot\right\|_{p}+\left\|\left\{2^{j\left(s+\frac{1}{2}-\frac{1}{p}\right)}\left\|d_{j \cdot}\right\|_{p}\right\}_{j \geq 0}\right\|_{q}<\infty$.

Furthermore,

$$
\|f\|_{B_{p, q}^{s}} \sim\left\|s_{0} \cdot\right\|_{p}+\left\|\left\{2^{j\left(s+\frac{1}{2}-\frac{1}{p}\right)}\left\|d_{j \cdot}\right\|_{p}\right\}_{j \geq 0}\right\|_{q} .
$$

Before introducing Fano's Lemma, we need the notation of Kullback-Leilber distance [4]. Let $P$ and $Q$ with $P$ being absolutely continuous with respect to $Q$ (denoted by $P \ll Q$ ). Then the Kullback-Leilber distance is defined by

$$
K(P, Q):=\int_{p \cdot q>0} p(x) \ln \frac{p(x)}{q(x)} \mathrm{d} x,
$$

Where $p$ and $q$ are density functions of $P, Q$ respectively.

Lemma 1.2. (Fano's Lemma, [4]) Let $\left(\Omega, \aleph, P_{k}\right)$ be probability measurable spaces and $A_{k} \in \mathfrak{N}, k=0,1, \cdots, m$. If $A_{k} \cap A_{v}=\phi$ for $k \neq v$, then with $A^{c}$ standing for the complement of $A$ and $\kappa_{m}:=\inf _{0 \leq v \leq m} \frac{1}{m} \sum_{k \neq v} K\left(P_{k}, P_{v}\right)$,

$$
\sup _{0 \leq k \leq m} P_{k}\left(A_{k}^{c}\right) \geq \min \left\{\frac{1}{2}, \sqrt{m} \exp \left(-3 e^{-1}-\kappa_{m}\right)\right\} .
$$

By Lemma 1.1 and 1.2, we can show the following result:

Theorem 1.1. Let $f \in \tilde{B}_{r, q}^{s}(R, L)$ with $1 \leq r, q \leq \infty$, $1 \leq p<\infty$ and $s r>1$. If $f_{n}$ is an estimator of $f$ with $n$ i.i.d. random samples, then

$$
\sup _{f \in \tilde{B}_{r, q}^{s}(R, L)} E\left(\left\|f_{n}-f\right\|_{p}\right) \succ \max \left\{\left(\frac{\ln n}{n}\right)^{\frac{s-1 / r+1 / p}{2(s-1 / r)+1}}, n^{-\frac{s}{2 s+1}}\right\},
$$

where $\tilde{B}_{r, q}^{s}(R, L):=\left\{f \in B_{r, q}^{s}(R),\|f\|_{B_{r, q}^{s}} \leq L\right\}$ and $f$ has compact support $\}$; The notation $x \succ y$ means $x \geq C y$ with a constant $C$.

Remark 1.1. Note that

$$
\max \left\{\left(\frac{\ln n}{n}\right)^{\frac{s-1 / r+1 / p}{2(s-1 / r)+1}}, n^{-\frac{s}{2 s+1}}\right\}=\left(\frac{\ln n}{n}\right)^{\frac{s-1 / r+1 / p}{2(s-1 / r)+1}}
$$

for $r \leq \frac{p}{2 s+1}$ and for $r>\frac{p}{2 s+1}$

$$
\max \left\{\left(\frac{\ln n}{n}\right)^{\frac{s-1 / r+1 / p}{2(s-1 / r)+1}}, n^{-\frac{s}{2 s+1}}\right\}=n^{-\frac{s}{2 s+1}} .
$$

Then theorem 1.1 is a reformulate of the lower bound in (1.1). By using the idea of reference [5], we show this theorem in the next two sections.

\section{Proof of Theorem 1.1}

Firstly, we prove

$$
\sup _{f \in \tilde{B}_{r, q}^{s}(R, L)} E\left(\left\|f_{n}-f\right\|_{p}\right) \succ\left(\frac{\ln n}{n}\right)^{\frac{s-1 / r+1 / p}{2(s-1 / r)+1}} .
$$
and

One need construct $g_{k}$ such that $g_{k} \in \tilde{B}_{r, q}^{s}(R, L)$

$$
\sup _{k} E\left(\left\|f_{n}-g_{k}\right\|_{p}\right) \succ\left(\frac{\ln n}{n}\right)^{\frac{s-1 / r+1 / p}{2(s-1 / r)+1}} .
$$

Let $\varphi$ be a compactly supported, $t(t>s)$ regular and orthonormal scaling function, $\psi$ be the corresponding wavelet with $\operatorname{supp} \psi \subseteq[0, l), l \in N^{+}$. Here and after, 
$N^{+}$denotes the set of positive integers. Then there exists a compactly supported density function $g$ (i.e.

$g(x) \geq 0$ and $\left.\int g(x) \mathrm{d} x=1\right)$ satisfying

$$
g(x) \in B_{r, q}^{s}(R) \text { and }\left.g(x)\right|_{[0, l]}=c_{0}>0 .
$$

Let $\Delta_{j}:=\left\{0, l, 2 l, \cdots,\left(2^{j}-1\right) l, 2^{j} l\right\}$. Then the number of elements in $\Delta_{j}$ is $2^{j}+1$, denoted by $\# \Delta_{j}=2^{j}+1$. Motivated by [5], one defines $a_{j}:=2^{-j(s+1 / 2-1 / r)^{j}}$ and

$$
g_{k}(x):=g(x)+a_{j} \psi_{j k}(x) I_{\left\{k \neq 2^{j} l\right\}}, k \in \Delta_{j}
$$

with $I_{\left\{k \neq 2^{j} l\right\}}:=1$ if $k \neq 2^{j} l$, else $I_{\left\{k \neq 2^{j} l\right\}}:=0$. Obviously, $g_{2^{j} l}=g, \int g(x) \mathrm{d} x=1$ and

$g_{k}(x) \geq c_{0}-2^{-j(s-1 / r)}\|\psi\|_{\infty} \geq 0$ for large $j$, which implies that $g_{k}$ is a density function for each $k$.

By the assumptions of $\varphi$, the wavelet $\psi$ is compactly supported and $t$ times differentiable. Therefore, $\psi \in B_{r, q}^{s}(R)(t>s)$ and $g_{k} \in \tilde{B}_{r, q}^{s}(R)$. Because $a_{j} 2^{j(s+1 / 2-1 / r)}=1,\left\|a_{j} \psi_{j k}\right\|_{B_{r, q}^{s}} \leq C$ and so is $\left\|g_{k}\right\|_{B_{r, q}^{s}}$ due to Lemma 1.1. Hence, $g_{k}(x) \in \tilde{B}_{r, q}^{s}(R, L)$. Clearly,

$$
\begin{aligned}
\left\|g_{k}-g_{k^{\prime}}\right\|_{p} & \geq\left\|g_{k}-g_{2^{j} l}\right\|_{p}=\left\|a_{j} \psi_{j k}\right\|_{p} \\
& =\|\psi\|_{p} 2^{-j(s+1 / p-1 / r)}:=\delta_{j}
\end{aligned}
$$

For $k \neq k^{\prime} \in \Delta_{j} /\left\{2^{j} l\right\}$ due to $a_{j}:=2^{-j(s+1 / 2-1 / r)}$. Furthermore, $A_{k}:=\left\{\left\|f_{n}-g_{k}\right\|_{p}<\frac{\delta_{j}}{2}\right\}$ satisfies $A_{k} \cap A_{k^{\prime}}=\phi$ for $k \neq k^{\prime}$. Recall that $\# \Delta_{j}=2^{j}+1$. By Lemma 1.2, $\sup _{k \in \Delta_{j}} P_{g_{k}}^{n}\left(A_{k}^{c}\right) \geq \min \left\{\frac{1}{2}, \sqrt{2^{j}} \exp \left(-\kappa_{2^{j}}-\frac{3}{e}\right)\right\}$. Here and after, $P_{f}^{n}$ stands for the probability measure corresponding to the density function $f^{n}(x):=f\left(x_{1}\right) f\left(x_{2}\right) \cdots f\left(x_{n}\right)$. It is easy to see that $P_{g_{k}}^{n} \ll P_{g_{0}}^{n}$ from the constructions of $g_{k}$. Since $f_{n}$ is an estimator of density with $n$ i.i.d. random samples,

$$
E\left(\left\|f_{n}-g_{k}\right\|_{p}\right) \geq \frac{\delta_{j}}{2} P_{g_{\varepsilon^{i}}}^{n}\left(\left\|f_{n}-g_{k}\right\|_{p} \geq \frac{\delta_{j}}{2}\right)=\frac{\delta_{j}}{2} P_{g_{k}}^{n}\left(A_{k}^{c}\right) .
$$

Then,

$$
\begin{aligned}
\sup _{k \in \Delta_{j}} E\left(\left\|f_{n}-g_{k}\right\|_{p}\right) & \geq \sup _{k \in \Delta_{j}} \frac{\delta_{j}}{2} P_{g_{k}}^{n}\left(A_{k}^{c}\right) \\
& \geq \frac{\delta_{j}}{2} \min \left\{\frac{1}{2}, \sqrt{2^{j}} \exp \left(-\kappa_{2^{j}}-\frac{3}{e}\right)\right\} .
\end{aligned}
$$

Next, one shows $\kappa_{2^{j}} \leq c_{0}^{-1} n a_{j}^{2}$ : Recall that

$$
\begin{aligned}
& K\left(P_{1}^{n}, P_{2}^{n}\right):=\int_{f_{1}^{n} \cdot f_{2}^{n}>0} f_{1}^{n}(x) \ln \frac{f_{1}^{n}(x)}{f_{2}^{n}(x)} \mathrm{d} x, \quad f_{1}^{n}(x)=\prod_{j=1}^{n} \\
& f_{1}\left(x_{j}\right) \text { and } f_{2}^{n}(x)=\prod_{j=1}^{n} f_{2}\left(x_{j}\right) \text {. Then } \\
& K\left(P_{1}^{n}, P_{2}^{n}\right)=\sum_{i=1}^{n} \int f_{1}\left(x_{i}\right) \ln \frac{f_{1}\left(x_{i}\right)}{f_{2}\left(x_{i}\right)} \mathrm{d} x_{i}=n K\left(P_{1}^{1}, P_{2}^{1}\right) .
\end{aligned}
$$

Note that $K\left(P_{1}^{1}, P_{2}^{1}\right):=\int f_{1}(x) \ln \frac{f_{1}(x)}{f_{2}(x)} \mathrm{d} x$ and $\ln u \leq u-1$ for $u>0$. Then

$$
\begin{aligned}
K\left(P_{1}^{n}, P_{2}^{n}\right) & =n \int f_{1}(x) \ln \frac{f_{1}(x)}{f_{2}(x)} \mathrm{d} x \\
& \leq n \int f_{1}(x)\left[\frac{f_{1}(x)}{f_{2}(x)}-1\right] \mathrm{d} x \\
& =n \int\left|f_{2}(x)\right|^{-1}\left|f_{1}(x)-f_{2}(x)\right|^{2} \mathrm{~d} x .
\end{aligned}
$$

Hence,

$$
\kappa_{2^{j}}:=\inf _{v \in \Delta_{j}} \sum_{k \neq \nu} 2^{-j} K\left(P_{g_{k}}^{n}, P_{g_{v}}^{n}\right) \leq 2^{-j} \sum_{k \in \Delta_{j}} K\left(P_{g_{k}}^{n}, P_{g_{2} j_{l}}^{n}\right) .
$$

Moreover,

$$
\kappa_{2^{j}} \leq 2^{-j} n \sum_{k \in \Delta_{j}} \int|g(x)|^{-1}\left|g_{k}(x)-g(x)\right|^{2} \mathrm{~d} x .
$$

According to the definition of $g_{k}$, $\operatorname{supp}\left(g_{k}-g\right) \subseteq[0, l]$ and $g(x)=c_{0}$ on $[0, l]$. Thus, $\int|g(x)|^{-1}\left|g_{k}(x)-g(x)\right|^{2}$ $d x=c_{0}^{-1} \int\left|a_{j} \psi_{j k}(x)\right|^{2} \mathrm{~d} x=c_{0}^{-1} a_{j}^{2}\left\|\psi_{j k}(x)\right\|_{2}^{2}=c_{0}^{-1} a_{j}^{2}$ by the orthonormality of $\psi_{j k}$. Then (2.3) reduces to

$$
\kappa_{2^{j}} \leq c_{0}^{-1} n a_{j}^{2} .
$$

Take $2^{j} \sim\left(\frac{n}{\ln n}\right)^{\frac{1}{2(s-1 / r)+1}}$. Then $n a_{j}^{2}=n 2^{-2 j\left(s+\frac{1}{2}-\frac{1}{r}\right)} \sim \ln n$. Now, one can choose $C>0$ such that $n a_{j}^{2} \leq C \ln n$ and $C[4(s-1 / r)+2]<c_{0}$. Therefore,

$$
\sqrt{2^{j}} e^{-\kappa_{2} j} \geq \sqrt{2^{j}} e^{-c_{0}^{-1}} n a_{j}^{2} \succ\left(\frac{n}{\ln n}\right)^{[4(s-1 / r)+2]^{-1}} n^{-C c_{0}^{-1}} \geq 1
$$

and (2.2) reduces to $\sup _{k \in \Delta_{j}} E\left(\left\|f_{n}-g_{k}\right\|_{p}\right) \geq C \delta_{j}$. Then the desired follows from $\delta_{j}=\|\psi\|_{p} 2^{-j(s+1 / p-1 / r)}$ by (2.1) and $2^{j} \sim\left(\frac{n}{\ln n}\right)^{\frac{1}{2(s-1 / r)+1}}$.

Now, we prove $\sup _{f \in \tilde{S}_{r, q}^{s}(R, L)} E\left(\left\|f_{n}-f\right\|_{p}\right) \succ n^{-\frac{s}{2 s+1}}$. Our proof depends on another lemma [4]. 
Lemma 2.1. (Varshamov-Gilbert) Let

$\Theta:=\left\{\varepsilon=\left(\varepsilon_{1}, \cdots, \varepsilon_{m}\right)\right\}, \quad \varepsilon_{i} \in\{0,1\}$. Then there exists a subset $\left\{\varepsilon^{0}, \cdots, \varepsilon^{M}\right\}$ of $\Theta$ with $\varepsilon^{0}=(0, \cdots, 0)$ such that $M \geq 2^{m / 8}$ and $\sum_{k=1}^{m}\left|\varepsilon_{k}^{i}-\varepsilon_{k}^{j}\right| \geq \frac{m}{8}(0 \leq i \neq j \leq M)$.

It is sufficient to construct $g_{\varepsilon^{i}}(i=0,1, \cdots, M)$ such that $g_{\varepsilon^{i}} \in \tilde{B}_{r, q}^{s}(R, L)$ and

$$
\sup _{i} E\left(\left\|f_{n}-g_{\varepsilon^{i}}\right\|_{p}\right) \succ n^{-\frac{s}{2 s+1}} .
$$

As proved above, let $\varphi$ be a compactly supported, $t(t>s)$ regular and orthonormal scaling function, $\psi$ be the corresponding wavelet with supp $\psi \subseteq[0, l), l \in N^{+}$. Assumes $g \in \tilde{B}_{r, q}^{s}(R, L)$ and $\left.g\right|_{[0, l]}=c_{0}>0$. Define $a_{j}:=2^{-j(s+1 / 2)}, \Delta_{j}:=\left\{0, l, 2 l, \cdots,\left(2^{j}-1\right) l\right\}$ and

$$
g_{\varepsilon}(x):=g(x)+a_{j} \sum_{k \in \Delta_{j}} \varepsilon_{k} \psi_{j k}(x)
$$

with $\varepsilon=\left(\varepsilon_{k}\right)_{k \in \Delta_{j}} \in\{0,1\}^{2^{j}}$ (note that $g_{0}=g$ ). Since $\varepsilon_{k} \in\{0,1\}$, one knows that $\sum_{k \in \Delta_{j}}\left|\varepsilon_{k}\right|^{r} \leq 2^{j}$ and

$$
2^{j(s+1 / 2-1 / r)} a_{j}\left(\sum_{k \in \Delta_{j}}\left|\varepsilon_{k}\right|^{r}\right)^{1 / r} \leq 1 .
$$

By Lemma 1.1, $\left\|a_{j} \sum_{k \in \Delta_{j}} \varepsilon_{k} \psi_{j k}\right\|_{B_{r, q}^{s}} \leq C$, and so is $\left\|g_{\varepsilon}\right\|_{B_{r, q}^{s}}$. Hence $g_{\varepsilon} \in \tilde{B}_{r, q}^{s}(R, L)$.

Note that the supports of $\psi_{j k}$ for $k \in \Delta_{j}$ are mutually disjoint. Then

$$
g_{\varepsilon}(x) \geq c_{0}-a_{j}\left\|\psi_{j k}\right\|_{\infty} \geq c_{0}-2^{-j s}\|\psi\|_{\infty} \geq 0
$$

for big $j$. This with $\int g_{\varepsilon}(x) \mathrm{d} x=\int g(x) \mathrm{d} x=1$ implies that $g_{\varepsilon}$ is a density function for each $\varepsilon \in\{0,1\}^{2^{j}}$. According to Lemma 2.1, there exists $\left\{\varepsilon^{0}, \varepsilon^{1}, \cdots, \varepsilon^{M}\right\}$ such that $M \geq 2^{2^{j-3}}$ and

$$
\sum_{k \in \Delta_{j}}\left|\varepsilon_{k}^{l}-\varepsilon_{k}^{i}\right| \geq 2^{j-3}
$$

Because supp $\psi_{j k} \cap \operatorname{supp} \psi_{j k^{\prime}}=\phi$ for $k \neq k^{\prime} \in \Delta_{j}$, one knows that

$$
\begin{aligned}
\left\|g_{\varepsilon^{l}}-g_{\varepsilon^{i}}\right\|_{p}^{p} & =\sum_{k \in \Delta_{j}} a_{j}^{p}\left|\varepsilon_{k}^{l}-\varepsilon_{k}^{i}\right|^{p}\left\|\psi \psi_{j k}\right\|_{p}^{p} \\
& =2^{-(s p+1) j}\|\psi\|_{p}^{p} \sum_{k \in \Delta_{j}}\left|\varepsilon_{k}^{l}-\varepsilon_{k}^{i}\right|^{p}
\end{aligned}
$$

This with (2.6) and $\varepsilon_{k}^{l}, \quad \varepsilon_{k}^{i} \in\{0,1\}$ leads to

$$
\begin{aligned}
& \left\|g_{\varepsilon^{l}}-g_{\varepsilon^{i}}\right\|_{p}^{p} \geq\|\psi\|_{p}^{p} 2^{-s p j} 2^{-3} \text { and } \\
& \qquad\left\|g_{\varepsilon^{l}}-g_{\varepsilon^{i}}\right\|_{p} \geq 8^{-1 / p}\|\psi\|_{p} 2^{-s j}:=\delta_{j} .
\end{aligned}
$$

Clearly, the sets $A_{\varepsilon^{i}}=\left\{\left\|f_{n}-g_{\varepsilon^{i}}\right\|<\frac{\delta_{j}}{2}\right\}(i=0,1, \cdots, M)$ satisfy $A_{\varepsilon^{l}} \cap A_{\varepsilon^{i}}=\phi$ for $i \neq l$. Then Fano's Lemma yields

$$
\sup _{0 \leq i \leq M} P_{g_{\varepsilon^{i}}}^{n}\left(A_{\varepsilon^{i}}^{c}\right) \geq \min \left\{\frac{1}{2}, \sqrt{M} \exp \left(-\kappa_{M}-3 e^{-1}\right)\right\} .
$$

On the other hand, it follows $\kappa_{M} \leq c_{0}^{-1} n a_{j}^{2} 2^{j}$ from the similar arguments to the proof of (2.4). Take

$2^{j} \sim n^{\frac{1}{2 s+1}}$. Then $n a_{j}^{2}=a 2^{-(2 s+1) j} \sim 1$. Hence, one can choose a constant $C>0$ such that

$$
\sqrt{M} e^{-\kappa_{M}} \geq 2^{2^{j-4}} \mathrm{e}^{-c_{0}^{-1} n a_{j}^{2} 2^{j}} \geq 2^{2^{j-4}} \mathrm{e}^{-c_{0}^{-1} C 2^{j}} \geq 1 .
$$

Therefore, (2.8) reduces to $\sup _{0 \leq i \leq M} P_{\varepsilon_{\varepsilon}}^{n}\left(A_{\varepsilon^{i}}^{c}\right) \geq C>0$ and

$$
\begin{aligned}
& \sup _{0 \leq i \leq M} E\left(\left\|f_{n}-g_{\varepsilon^{i}}\right\|_{p}\right) \\
& \geq \sup _{0 \leq i \leq M} \frac{\delta_{j}}{2} P_{g_{\varepsilon^{i}}}^{n}\left(\left\|f_{n}-g_{\varepsilon^{i}}\right\|_{p} \geq \frac{\delta_{j}}{2}\right) \geq C \delta_{j} .
\end{aligned}
$$

This with (2.7) and $2^{j} \sim n^{\frac{1}{2 s+1}}$ yield (2.5).

\section{Acknowledgements}

The author Huiying Wang is grateful to the referees for their valuable comments and thanks her advisor, Professor Youming Liu, for his helpful guidance. This work is supported by the National Natural Science Foundation of China (No. 10871012) and Natural Science Foundation of Beijing (No. 1082003).

\section{References}

[1] G. Kerkyacharian and D. Picard, "Density Estimation in Besov Spaces,” Statistics \& Probability Letters, Vol. 13, No. 1, 1992, pp. 15-24. doi:10.1016/0167-7152(92)90231-S

[2] D. L. Donoho, I. M. Johnstone, G. Kerkyacharian and D. Picard, "Density Estimation by Wavelet Thresholding," The Annals of Statistics, Vol. 24, No. 2, 1996, pp. 508539. doi:10.1214/aos/1032894451

[3] W. Härdle, G. Kerkyacharian, D. Picard and A. B. Tsybakov, "Wavelets, Approximation and Statistical Applications,” Springer-Verlag, New York, 1997.

[4] A. B. Tsybakov, "Introduction to Nonparametric Estimation,” (English) Revised and Extended from the 2004 French Original, Translated by Vladimir Zaiats, Springer Series in Statistics, Springer, New York, 2009.

[5] P. Baldi, G. Kerkyacharian, D. Marinucci and D. Picard, "Adaptive Density Estimation for Directional Data Using Needlets," The Annals of Statistics, Vol. 37, No. 6A, 
2009, pp. 3362-3395. doi:10.1214/09-AOS682

[6] C. Christophe, "Regression with Random Design: A Minimax Study,” Statistics \& Probability Letters, Vol. 77, No. 1, 2007, pp. 40-53. doi:10.1016/j.spl.2006.05.010
[7] A. B. Tsybakov, “Optimal Rates of Aggregation,” COLT/ Kernel 2003 Lecture Notes in Artificial Intelligence 2777, Springer, Heidelberg, 2003, pp. 303-313. 